2004

\title{
Direct observation of microparticle gyromotion in a magnetized direct current glow discharge dusty plasma
}

William E. Amatucci

David N. Walker

George Gatling

Earl E. Scime

Follow this and additional works at: https://researchrepository.wvu.edu/faculty_publications

\section{Digital Commons Citation}

Amatucci, William E.; Walker, David N.; Gatling, George; and Scime, Earl E., "Direct observation of microparticle gyromotion in a magnetized direct current glow discharge dusty plasma" (2004). Faculty Scholarship. 130.

https://researchrepository.wvu.edu/faculty_publications/130 


\title{
Direct observation of microparticle gyromotion in a magnetized direct current glow discharge dusty plasma
}

\author{
William E. Amatucci and David N. Walker \\ Plasma Physics Division, Naval Research Laboratory, Washington, DC 20375 \\ George Gatling \\ Advanced Technology Division, SFA Incorporated, Largo, Maryland 20774 \\ Earl E. Scime \\ Department of Physics, West Virginia University, Morgantown, West Virginia 26506
}

(Received 20 October 2003; accepted 20 January 2004; published online 16 April 2004)

\begin{abstract}
Laboratory observations of oscillatory motion of charged microparticles have been made in an argon dc glow discharge plasma created within a strong dc magnetic field. Measurements of the oscillation frequency and amplitude are consistent with the gyromotion of magnetized dust grains under the ambient plasma conditions. The measurements provide an effective method for the noninvasive determination of the charge on the observed microparticles. The observations also seem to indicate that the neutral drag force on the dust grains may be smaller than anticipated from the classical estimation. (C) 2004 American Institute of Physics. [DOI: 10.1063/1.1669396]
\end{abstract}

\section{INTRODUCTION}

The magnitude of charge on dust particles determines the properties of a dusty plasma. The process by which dust grains become charged depends upon environmental factors. For example, in space, dust grains are usually charged by the collected flux of charged plasma particles, by photoionization due to incident UV radiation, or by secondary electron emission. In the laboratory, charging is primarily due to charged particle collection, with the dust typically becoming negatively charged. It is generally believed that the dust grains act much like isolated probes and charge to the local floating potential in the plasma. Since the charged microparticles also have appreciable mass, gravitational forces, as well as electrical, magnetic, radiation, and ion and neutral drag forces all can affect the particle motion.

Because of its fundamental importance, a number of previous experimental investigations have focused on the measurement of dust grain charge. For example, microparticle charge has been measured by collecting dust grains that fall through the plasma into a Faraday cup. ${ }^{1}$ However, those measurements involved the removal of the particles from the system and whether the grains retain the same value of charge outside the plasma as they possess inside remains an important unanswered question. In other experiments, the charge $Q \equiv Z e$ has been measured while the particles remained in the plasma. These techniques involved laserexcited resonances ${ }^{2}$ in a rf discharge plasma and analysis of particle oscillations about a plasma sheath. ${ }^{3}$ Charge measurements have also been performed in rf discharge plasmas by an analysis of the compression of a two-dimensional (2-D) plasma crystal layer under the influence of a known transverse confining force. ${ }^{4}$ While this is a sensitive method for the determination of charge, it does require the particles to be in a crystalline state and also is dependent on a model of the crystallization process.
In nearly all dusty plasma experiments, a magnetic field is either absent entirely or else it affects only the ions and electrons. In either case, the charged dust is treated as an unmagnetized component. However, there have been several experimental investigations into the role of magnetic fields on dusty plasmas. Sato et al. ${ }^{5}$ performed experiments in a Q-machine potassium plasma which also included $\mathrm{C}_{60}$ "Bucky balls." The 0.7-nm diameter particles, with a mass number of 720, each attached only one electron in the plasma. The authors regarded these as heavy negative ions, but they could likewise be considered a fine dust material. To determine the charge on the $\mathrm{C}_{60}$ particles, the authors used an omegatron spectrum analyzer, in which collected particle current is analyzed as a function of frequency of an applied rf electric field. A clear spectral peak at the cyclotron frequency for singly charged $\mathrm{C}_{60}$ particles was found. In addition, the authors investigated low-frequency wave modes excited by applying a positive bias to a small disk electrode contained within the plasma column. In this case, the familiar current-driven electrostatic ion cyclotron instability ${ }^{6}$ was observed for the potassium ions, but a new spectral feature was also detected which appeared to be consistent with the $\mathrm{C}_{60}$ cyclotron frequency. Together, these experiments provide evidence for the magnetization of the small singly charged $\mathrm{C}_{60}$ particles.

In a different series of Q-machine experiments in which nanometer-sized dust grains were dropped through the plasma column, Luo and D'Angelo ${ }^{7}$ monitored the electron density as a function of time after the dust dispenser was stopped. With the dust dispenser turned on, it was found that virtually all of the electrons resided on the dust grains. The typical charge on the $1-5 \mathrm{~nm}$ grains was estimated to be $1-4$ electron charges. When the dust dispenser was turned off, it was found that, depending upon plasma density, magnetic field strength and geometry, the time it took for the electron population to recover could vary from a few seconds to more 


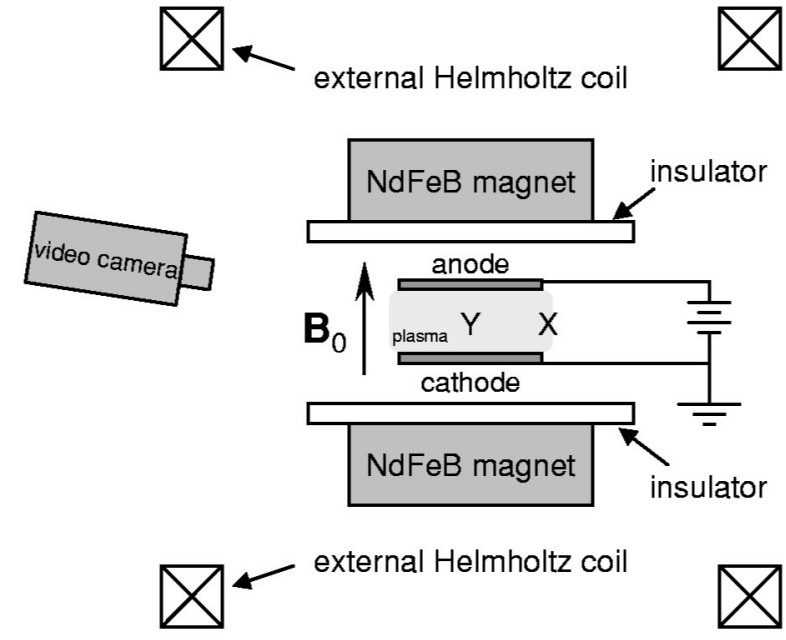

FIG. 1. A schematic diagram of the experimental setup as viewed from the position of the laser used to illuminate the charged dust particles.

than $100 \mathrm{~s}$. The authors concluded that the cases of delayed electron density recovery could be plausibly explained by the magnetic confinement of small dust grains.

While each of these experiments provide indirect evidence of the magnetization of nanometer-sized dust particles that hold only one to a few electrons, direct observation of the particle motion was not possible. The experiments described in this paper focus on the effects of a magnetic field strong enough that micron-sized dust is magnetized. The effect of a strong magnetic field on the charging and motion of micron-sized dust grains with $Z \sim 10^{4}$ in a plasma is examined. We report direct observations of dust cyclotron motion and demonstrate how the measurements can be used for the sensitive noninvasive determination of dust grain charge, $Z$.

\section{EXPERIMENTAL SETUP}

The experiments reported here were performed in the Naval Research Laboratory's newly constructed DUsty PLasma EXperiment (DUPLEX Jr.), a transparent polycarbonate cylindrical vacuum chamber that is $46 \mathrm{~cm}$ in diameter and $61 \mathrm{~cm}$ in height. An aluminum plate with five vacuum flanges provides probe access to the experimental volume from the top. Langmuir and emissive probes are used to measure the floating potential $V_{f}$ and the plasma potential $V_{p}$, respectively. The polycarbonate cylinder sits upon a stainless steel chamber section that provides access to a 4 in. diffusion pump-based vacuum system. The base pressure of the system is approximately $1 \times 10^{-5}$ Torr.

Argon dc glow discharge plasmas were created for these experiments. A schematic diagram of the experimental setup is shown in Fig. 1. The anode and cathode were constructed from 1.75 in.-diameter by $1 / 8$ in.-thick mirror-polished stainless steel. The anode was biased positive with respect to the grounded cathode. The anode and cathode were separated by a distance of $2 \mathrm{~cm}$ and were centered axially between a pair of neodymium-iron-boride permanent magnets that provided a strong axial magnetic field. The magnets are 1 in. thick by $2 \mathrm{in}$. wide by $3 \mathrm{in}$. long. The magnetic field is aligned with the 1-in. dimension. Unless otherwise noted, the magnetic field strength at the location of the particle observations was $2.5 \mathrm{kG}$. The direction of the magnetic field can be controlled by the orientation of the magnets and points either vertically upward or downward along the anode-cathode cylindrical axis. Across the experimental region, the magnetic field is reasonably uniform and can be varied up to approximately 5 $\mathrm{kG}$ by adjusting the magnet spacing. A pair of Helmholtz electromagnet coils (24 in. inside diameter) located outside the vacuum vessel and centered on the anode-cathode pair allow the magnetic field in the experimental region to be varied by $\pm 75 \mathrm{G}$.

Alumina microparticles (Alfa Aesar Stock \#39814) were used for these experiments. The Gaussian distributed particles are nominally $1.2 \pm 0.5 \mu \mathrm{m}$ diameter. The mass of a 1.2 $\mu \mathrm{m}$ particle is $\sim 2.5 \times 10^{-16} \mathrm{~kg}$. A small amount of the alumina dust was placed directly on the cathode. Once the discharge was initiated, individual dust grains charge up to the floating potential and levitate within the plasma. For the observations reported in this paper, the individual dust grains observed were found approximately 1 centimeter above the cathode and can be radially located anywhere from the center of the discharge to slightly beyond the outer diameter of the cathode.

\section{OBSERVATIONS}

With a discharge voltage of $230 \mathrm{~V}$ and a neutral argon pressure of 220 mTorr, several distinct regions were found where individual particles were separated well enough from other suspended particles for their motion to be observed unobstructed. For the initial observation to be described the magnetic field direction was upward and the position where the particles were found is indicated by the " $\mathrm{X}$ " in Fig. 1, above and slightly to the right of the cathode. The plasma density was approximately $10^{10} \mathrm{~cm}^{-3}$ and the electron temperature was $\sim 1.6 \mathrm{eV}$.

The suspended dust particles were illuminated by a 20-mW 670-nm diode laser. The beam spot is elliptical and was approximately 3 -mm wide and 1-mm thick at the location of the particles. The beam was oriented at an angle of $90^{\circ}$ with respect to the camera, which corresponds to the direction facing into the page in Fig. 1. The laser was aligned such that the wide dimension of the beam was oriented perpendicular to the magnetic field direction. The beam could be spread into a sheet with a cylindrical lens, but it was found that the natural width of the beam was sufficient to follow the particle motion and that individual grains were much more visible with the unspread beam.

Uniform circular motion of individual particles in a plane perpendicular to the magnetic field was recorded using a CCD imager operating at 30 frames per second. A composite image showing the position of a particle designated as Particle A at various points during two periods of the orbit is shown in Fig. 2. The image was made using every third video frame. This particular particle was observed to oscillate for more than 5 minutes before being perturbed by other dust grains moving nearby. A plot of the particle's position as a function of time for a period of one minute is shown in Fig. 


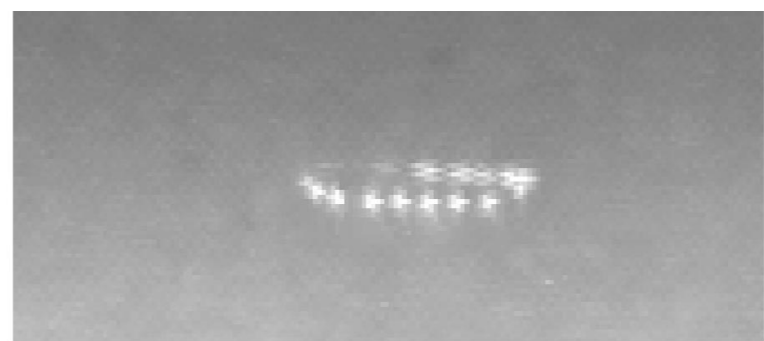

FIG. 2. Composite image of orbiting dust particle labeled Particle A. The image was formed from selected video frames taken during two oscillation periods.

3(a), while Fig. 3(b) shows an expanded view of five seconds of the time series. The particle motion is clearly periodic. The orbital period is $1.12 \pm 0.02$ seconds and the mean oscillation amplitude is $1.57 \pm 0.07 \mathrm{~mm}$. The time derivative of the Particle A position data indicates that the peak transverse velocity is $\sim 8.3 \mathrm{~mm} / \mathrm{s}$. Figure 3(c) shows the Fourier transform of the position time series data. A clear narrow peak at a frequency of $0.89 \mathrm{~Hz}$ corresponding to the particle oscillation is visible.

Figures 4(a) and 4(b) show sections of the time series of two additional examples of such periodic motion. The particle illustrated in Fig. 4(a), designated as Particle B, had an oscillation frequency of $0.83 \mathrm{~Hz}$ and was followed for over one minute before the particle was lost. Its orbital radius was $0.35 \mathrm{~mm}$ and the peak transverse velocity was $2.1 \mathrm{~mm} / \mathrm{s}$. Higher oscillation frequencies have also been observed as illustrated by the motion of Particle C shown in Figure 4(b). This particle was followed for over 2 minutes and $15 \mathrm{sec}-$
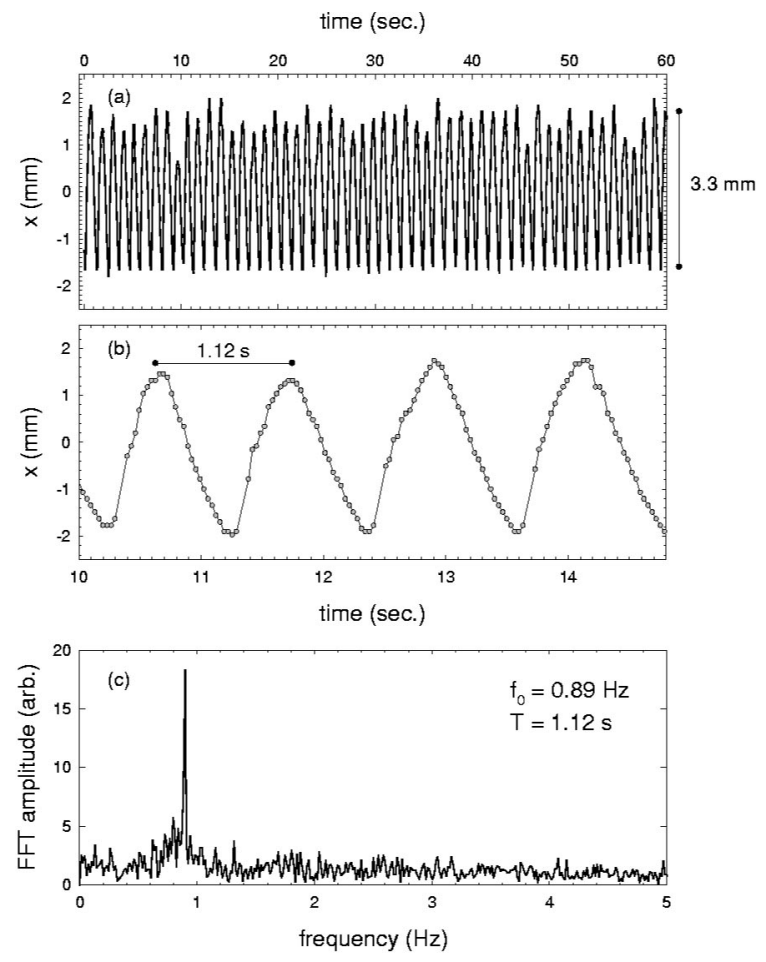

FIG. 3. (a) One minute of the Particle A horizontal position as a function of time data. (b) Enlarged view showing details of several orbital periods. (c) Fourier transform of particle position time series data.

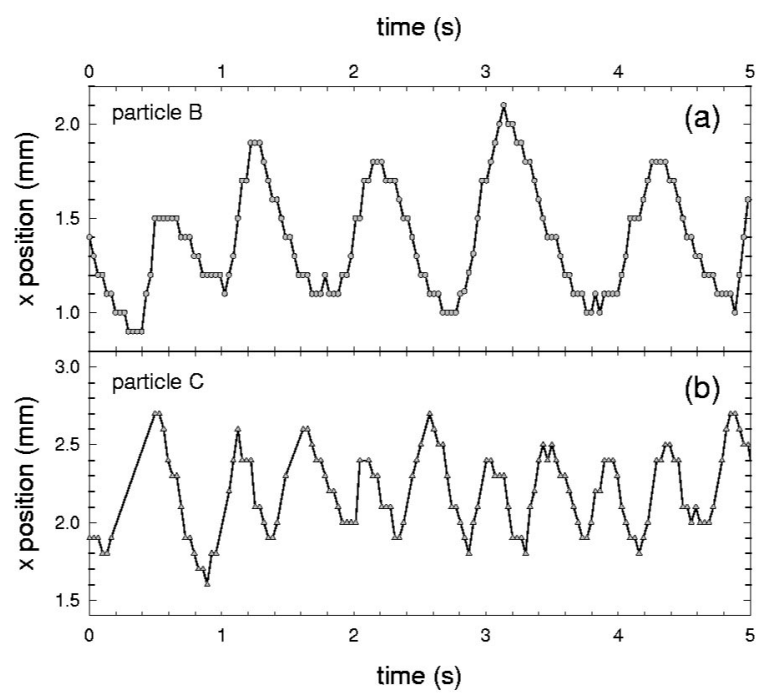

FIG. 4. (a) Horizontal position versus time data for Particle B. (b) The Particle $\mathrm{C}$ position as a function of time.

onds and was observed to oscillate at a frequency of $1.9 \mathrm{~Hz}$. Its orbital radius was $0.35 \mathrm{~mm}$ and the peak transverse velocity was $2.8 \mathrm{~mm} / \mathrm{s}$.

\section{DISCUSSION}

To determine if the observed oscillatory motion is cyclotron motion, several factors must be considered. First, an estimate of the anticipated particle gyrofrequency and gyroradius must be made in order to determine if the observed orbital frequency and radius fall within the expected range for our distribution of particle sizes and ambient plasma conditions. Second, the direction of the particle rotation must be determined for comparison to the expected direction of cyclotron motion for a negatively charged particle. Third, the values of oscillation frequency and orbital radius must be measured as functions of magnetic field strength and perpendicular velocity, respectively, in order to test for the appropriate scalings expected for cyclotron motion. Finally, an analysis of the forces acting on the particle and of alternate possible explanations is necessary to check for consistency between gyromotion and the laboratory observations.

\section{A. Estimation of dust particle gyrofrequency and gyroradius}

To estimate the gyrofrequency for a typical alumina particle in this experiment, we first assume that the particles are roughly spherical with a radius $r_{d}=0.6 \mu \mathrm{m}$ (corresponding to the $1.2 \mu \mathrm{m}$ peak in the size distribution). In the plasma, the particles act much like spherical capacitors, acquiring a charge $Q=C V$. Therefore, the microparticle charge can be described by $Q=4 \pi \varepsilon_{0} r_{d} \Delta V$, where $\Delta V$ is the difference between the floating potential and the plasma potential $V_{f}-V_{p}$. Since the dust cyclotron frequency is given by $\Omega_{d}$ $=Q B / M$, we can re-express the gyrofrequency in terms of the particle radius as 


$$
\Omega_{d}=\frac{3 \varepsilon_{0} \Delta V B}{\rho r_{d}^{2}} .
$$

Here $\rho$ is the mass density of the dust grain. The measured values of $V_{p}=146 \mathrm{~V}$ and $V_{f}=75 \mathrm{~V}$ indicate that $\Delta V$ $=-71 \mathrm{~V}$. (The measurement of $\Delta V$ is described further in the Appendix.) Since $B=0.25 \mathrm{~T}$, Eq. (1) yields a value of $\Omega_{d}=4.7 \mathrm{rad} / \mathrm{s}$, or $f_{d}=0.75 \mathrm{~Hz}$. If particles with radii plus/ minus one standard deviation are considered, then the expected range of gyrofrequencies is $0.38 \mathrm{~Hz}$ to $2.2 \mathrm{~Hz}(2.4$ $\mathrm{rad} / \mathrm{s}$ to $13.8 \mathrm{rad} / \mathrm{s})$. The measured frequencies of Particles A $(0.89 \mathrm{~Hz}), \mathrm{B}(0.83 \mathrm{~Hz})$, and $\mathrm{C}(1.9 \mathrm{~Hz})$ fall within this range.

To estimate the expected particle gyroradius, given by $\rho_{d}=v_{\perp} / \Omega_{d}$, the perpendicular velocity must also be determined. The particle velocities are determined from the time derivative of the particle position data and generally range from $\sim 1 \mathrm{~mm} / \mathrm{s}$ to $\sim 10 \mathrm{~mm} / \mathrm{s}$. Assuming a $1.2 \mu \mathrm{m}$ particle suspended in a plasma with $\Delta V=-71 \mathrm{~V}$ and $B=0.25 \mathrm{~T}$, the expected gyroradii would range from $\sim 0.2 \mathrm{~mm}$ to $\sim 2 \mathrm{~mm}$. The orbital radii of Particles $\mathrm{A}, \mathrm{B}$, and $\mathrm{C}$ fall within this range. Assuming $1.2 \mu \mathrm{m}$ particles, the expected gyroradii for Particles A, B, and C determined using their measured perpendicular velocities are $1.75 \mathrm{~mm}, 0.44 \mathrm{~mm}$, and $0.59 \mathrm{~mm}$, respectively. These agree reasonably well with the experimentally measured values of $1.57 \mathrm{~mm}$ for Particle A, and $0.35 \mathrm{~mm}$ for both Particles B and C. It should be noted that the particle grain diameters may be slightly different than 1.2 $\mu \mathrm{m}$, which would change the expected value of both $\Omega_{d}$ and $\rho_{d}$.

\section{B. Rotation direction determination}

To be cyclotron motion, the charged particles must orbit in the diamagnetic direction. The determination of the rotational direction of the particles is difficult because of the low viewing angles in the experimental configuration. The viewing angle from the camera to the center of Particle A, B, and $\mathrm{C}$ orbits was approximately $8^{\circ}$. Since there is some slight curvature to the magnetic field lines at the edge of the cathode, one cannot assume that the plane of the particle orbit is parallel to that of the anode and cathode. In particular, because of the low viewing angle, the change in the projected $y$ position for a level orbit would only be approximately 5-6 pixels. Thus, conclusions regarding the direction of orbital motion made from the relative $x$ and $y$ particle positions may not be reliable. However, by taking advantage of the mirror finish on the cathode, steeper viewing angles can be obtained by examining the particle motion in reflection. The effective viewing angle of the particle reflection is $\sim 15^{\circ}$. Figure 5 shows a composite image of the orbiting particle and the corresponding reflection for Particle A. The arrow indicates the direction of motion of the particle's reflection. Figure 6 shows plots of the reflection $x$ and $y$ positions from the video images as a function of time. While only about half of the orbit appears because the reflection is located near the edge of the cathode, the position versus time data show that while moving from right to left (i.e., from larger $x$ pixel values to smaller values), the particle first moves upward in the $y$ position then downward (i.e., from larger $y$ values to smaller then to larger again). Since moving vertically upward in the

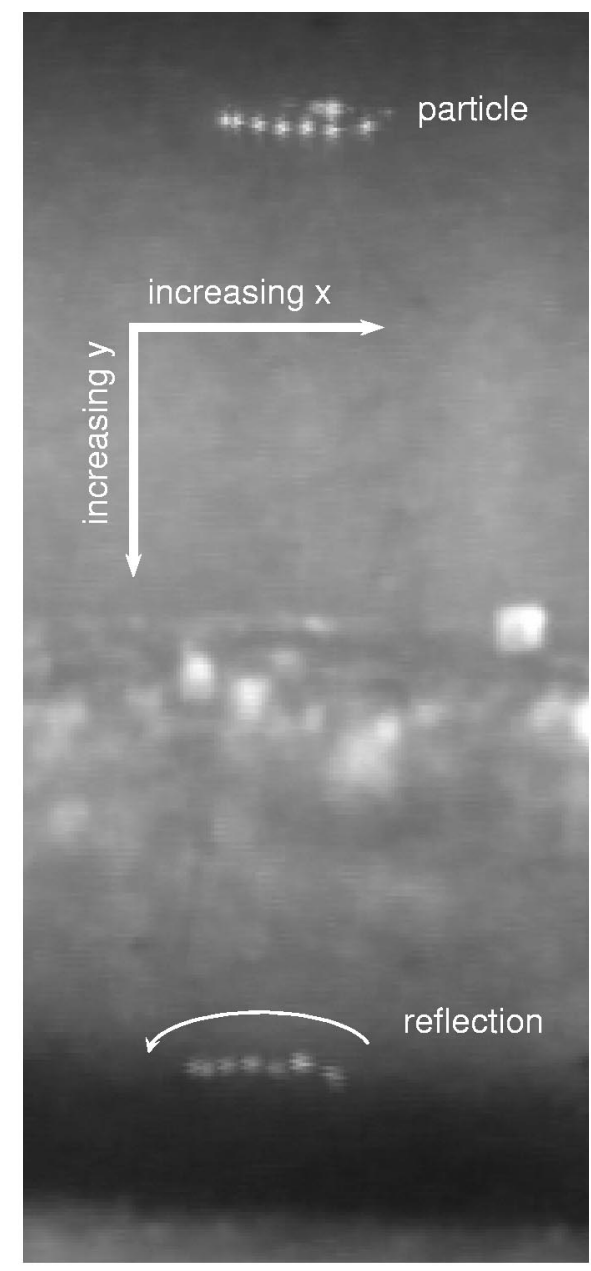

FIG. 5. Composite image of Particle A and its reflection off the mirrorfinished cathode. For this observation, the magnetic field is directed upward.

image corresponds to the reflection point moving away from the camera, these data indicate that the particle rotates in the counterclockwise sense if viewed from above. This is the expected direction of rotation of a negatively charged particle gyrating in an upward directed magnetic field.

To test that the orbital motion is consistent with the expected gyromotion direction, the direction of the magnetic field was reversed by turning over the permanent magnets. For these measurements, the platform holding the permanent magnet above the anode was modified to increase the direct

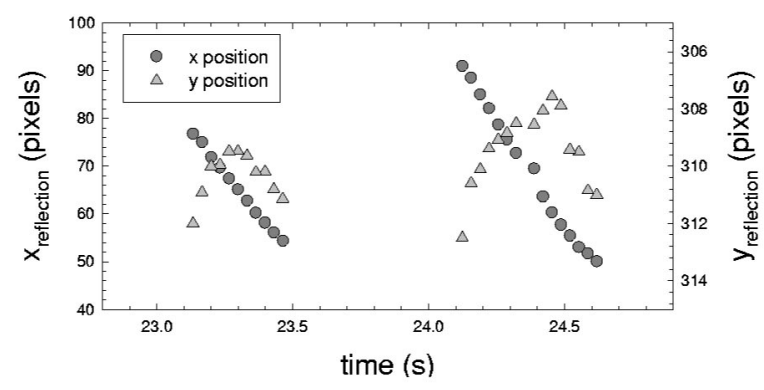

FIG. 6. Particle A reflection $x$ and $y$ position versus time data. 


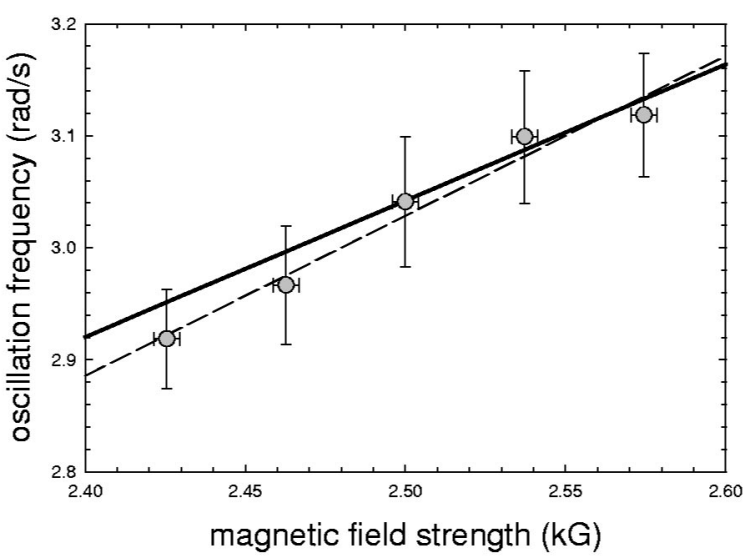

FIG. 7. Dust particle oscillation frequency as a function of magnetic field strength. The circles indicate the measured oscillation frequency and the dashed line is a least-squares linear fit to the data. The slope of the fit indicates a $Q / M$ value of 14.3. The solid line indicates the expected value of oscillation frequency as $B$ is varied for $Q / M=12.1$, which is determined by the measured oscillation frequency when there is no applied Helmholtz coil field.

viewing angle. In this case, with the magnetic field directed downward, the particles were observed to rotate in the clockwise direction and were located near the center of the cathode at the position indicated by the "Y" in Fig. 1. This again is consistent with the expected direction of gyrorotation for a negatively charged particle.

\section{Magnetic field and perpendicular velocity scaling}

The definitions of cyclotron frequency and gyroradius provide scalings that particles in cyclotron motion must follow. Since the dust particle gyrofrequency $\Omega_{d}=(Q / M) B$, it is expected that for a constant charge-to-mass ratio, the observed oscillation frequency should vary linearly with magnetic field strength. To test this property, a pair of Helmholtz electromagnet coils that are external to the vacuum vessel were used. The coils could provide an additional dc magnetic field of up to $75 \mathrm{G}$ directed either parallel or antiparallel to the $2.5 \mathrm{kG}$ field created by the $\mathrm{NdFeB}$ permanent magnets. While stronger fields could be created with the Helmholtz coils, the secondary field was intentionally kept small to minimize alterations to the ambient plasma parameters.

Figure 7 is a plot of the observed particle oscillation frequency as a function of magnetic field strength. For this experiment, the discharge voltage was $250 \mathrm{~V}$, the neutral pressure was $230 \mathrm{mTorr}$, and $\Delta V \approx-46 \mathrm{~V}$. To ensure uniform lighting of the particles over the entire diameter of the orbit, a 532-nm, 180-mW diode laser was used with the beam spread horizontally by a cylindrical lens. The data indicate that the oscillation frequency varies linearly with magnetic field strength. It is important to note that the same group of particles was followed throughout this experiment and that the height of the sheet of laser light used to illuminate the particles was not changed. Since the particles were all observed at the same vertical height above the cathode (i.e., $m g=Q E_{\|}$is satisfied at the same vertical location), it is reasonable to conclude that the charge-to-mass ratio is very

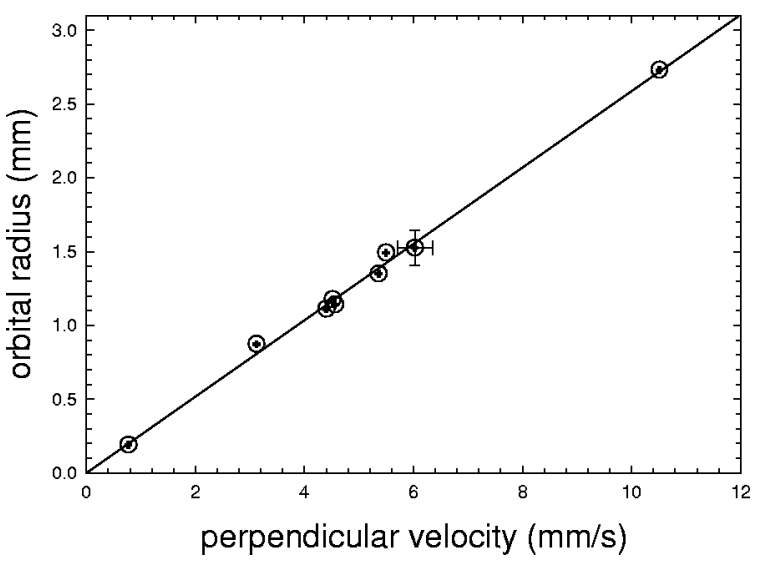

FIG. 8. The dust particle orbital radius as a function of tangential velocity. Orbital radii are plotted versus perpendicular velocity for individual particles observed in a group of oscillating dust grains.

nearly the same for all the particles and varies little throughout the experiment. The dashed line is a least squares fit to the measured oscillation frequencies (circles) and the slope is a measure of the particle charge-to-mass ratio. These data indicate a value of 14.3 for the charge-to-mass ratio. ${ }^{8}$ With no externally applied Helmholtz field, the Langmuir and emissive probe measurements of the plasma and floating potentials suggest a charge-to-mass ratio of 12.3 for a $1.2 \mu \mathrm{m}$ particle. Under the same conditions, the oscillation frequency was found to be $0.48 \mathrm{~Hz}(3.02 \mathrm{rad} / \mathrm{s})$, yielding $Q / M=12.1$, in good agreement with calculations based on the the probe measurements. Assuming that the charge-to-mass ratio remains constant, the value $Q / M=12.1$ can be used to predict the expected value of dust grain gyrofrequency as the magnetic field is changed, shown by the solid line in Fig. 7. Consistent with cyclotron motion, the data indicate that rotation frequency scales linearly with the magnetic field strength and that the slope is proportional to the charge-tomass ratio.

A second important cyclotron motion scaling is that of the particle gyroradius. For constant magnetic field strength and charge-to-mass ratio, the dust gyroradius $\rho_{d}$ $=M v_{\perp} / Q B$ varies linearly with perpendicular velocity. Figure 8 shows the measured orbital radius as a function of tangential velocity for individual dust grains found in a group of oscillating particles. For these data, the discharge voltage was $257 \mathrm{~V}$ and the neutral pressure was 280 mTorr. Langmuir and emissive probe measurements indicate that $V_{f}=143 \mathrm{~V}$ and $V_{p}=205 \mathrm{~V}$, yielding $\Delta V=-62 \mathrm{~V}$. Again, the particles were illuminated by a thin horizontal sheet of laser light that was not moved during the experiment, so the particles all have nearly identical charge-to-mass ratios. Thus, in comparing the orbital radii of the observed particles to the gyroradius, the coefficient $M / Q B\left(=\Omega_{d}^{-1}\right)$ can be taken to be constant for this group of particles. The data show that the orbital radius scales linearly with the tangential velocity, as expected for gyromotion. Furthermore, the reciprocal of the slope of a least-squares linear fit to the data gives a measure of the gyrofrequency. In this case, the slope indicates that $\Omega_{d}=3.88 \mathrm{rad} / \mathrm{s}$ and a charge-to-mass ratio of 15.5 . Using the probe measurements of $\Delta V$ and assuming a typical 


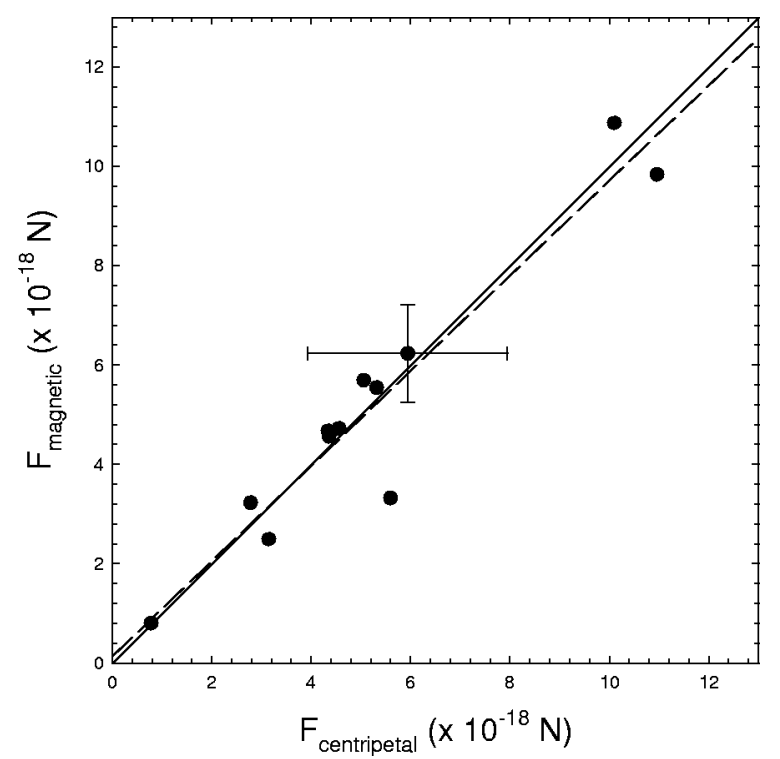

FIG. 9. A comparison of the centripetal and magnetic forces calculated using measurements of the orbital radius and tangential velocity from the video images and the probe measurements of the plasma and floating potentials. The dashed line is a least-squares fit to the data. The solid line is a reference line with slope equal to unity.

particle diameter of $1.2 \mu \mathrm{m}$, a value of $Q / M=16.6$ would be expected.

\section{Analysis of particle forces}

Cyclotron motion of a charged particle results from the centripetal acceleration provided by the Lorentz force, which acts when there is a component of the particle's velocity that is perpendicular to a magnetic field. Whether or not the motion described above is dust cyclotron motion, it is clearly uniform circular motion. Consequently, the centripetal force can be determined from the measurement of the particle's tangential velocity. Using Particle A as an example, the measurements indicate that the centripetal force required to maintain the uniform circular motion, $F_{c}=M v_{\perp}^{2} / r$ $=1.1 \times 10^{-17} \mathrm{~N}$. Using the plasma and floating potential measurements, it is found that the magnetic force $F_{M}$ $=Q v_{\perp} B=\left(4 \pi \varepsilon_{0} r_{d} \Delta V\right) v_{\perp} B=1.0 \times 10^{-17} \mathrm{~N}$, which compares favorably to the centripetal force. Figure 9 shows a comparison of the magnetic force to the centripetal force for the rest of the particles analyzed using the same method for determination of the forces. A perfect match between the centripetal and magnetic forces would yield a straight line with slope equal to unity, as shown by the solid line in Fig. 9. The dashed line is a least squares fit to the data, which has a slope of 0.958 . These data strongly suggest that the dust grain motion is controlled by the magnetic field.

While the data seem to be consistent with the expectations for cyclotron motion, other possible explanations should also be considered. One must consider the possibility that the particles could be trapped in small-scale potential wells within which the oscillatory motion takes place. In such a case, a localized radial electric field would provide the necessary centripetal force. If this were the case, to be consistent with the experimental observation that the orbital ra- dius scales linearly with the perpendicular velocity, the trapping potential well would have to be parabolic. In such a case, the radial electric field is proportional to $\left(\phi_{0} / 2 r_{0}^{2}\right) r$, where $\phi_{0}$ is the depth of the potential well and $r_{0}$ is the outer radius of the well. Setting the centripetal force equal to the electric force, one finds that the orbital radius is given by $r$ $=\left(M r_{0}^{2} / 2 Q \phi_{0}\right)^{1 / 2} v_{\perp}$. Therefore, using the radial electric field model to account for the particle rotation, it is found that the slope of the $r$ vs $v_{\perp}$ plot would be inversely proportional to the square root of the charge-to-mass ratio $\left[(Q / M)^{-1 / 2}\right]$, which is inconsistent with the experimental observation that the slope is proportional to $\Omega_{d}^{-1}$ $\sim(Q / M)^{-1}$. For such an electric field model to account for the observations would require the potential to have a specific value such that $2 \phi_{0} / r_{0}^{2}=Q / M$. Thus, we conclude that it is highly unlikely that electric fields due to small-scale potential wells are responsible for the observed motion.

Previously, a dc glow discharge plasma experiment investigating microparticle flows in the presence of a $400 \mathrm{G}$ magnetic field variable was reported. ${ }^{9}$ With the application of the field, an azimuthal rotation in the diamagnetic direction, but with rotation frequency much larger than the dust cyclotron frequency, was observed when the dust density was sufficiently high. This is in contrast to our observations, where individual isolated particles have been observed performing uniform circular motion at a frequency consistent with the dust gyrofrequency. The authors concluded that the motion was not gyromotion because the magnetic field strength was too weak to have a direct effect on the dust particle motion. They speculate that modifications to the potential profile caused by the effect of the magnetic field on the ions and electrons could drive the dust motion.

It has been shown that shear in the ion flow around a charged dust grain can lead to spinning of the particle with frequencies much higher than the dust cyclotron frequency. ${ }^{10}$ The magnetic moment created by this particle spin can lead to a precession of the dust grain with frequency slightly higher than the dust cyclotron frequency. However, since the particle motion observed in our experiments is associated with a finite gyroradius and is not simply a precession of the particle, this mechanism does not explain our observations. The observed orbital radii are consistent with a gyroradius $\rho_{d}=v_{\text {th }} / \Omega_{d}$, where $v_{\text {th }}$ is the dust thermal velocity and the dust is assumed to be in thermal equilibrium with the roomtemperature neutral gas. In such a case, the thermal speed of a $1.2 \mu \mathrm{m}$ particle would be $\sim 4 \mathrm{~mm} / \mathrm{s}$, consistent with the velocity measurements shown in Fig. 8.

Recently, orbiting particles in the horizontal plane in a capacitively coupled rf discharge plasma in the absence of a magnetic field have been reported. ${ }^{11}$ The dust particles used were $9.8 \mu \mathrm{m}$ in diameter, but the rotating particles observed were heavier than a single grain and were assumed to be agglomerates of particles. However, those experiments showed that different particles could orbit in both clockwise and counterclockwise fashion at the same time. Since there was an observed pressure dependence on the oscillation frequency, it was concluded that ion drag forces may be responsible for the observed behavior. In our case, there has been no observed dependence on neutral pressure and all particles 
were observed to rotate in the same directioncounterclockwise with $B$ directed upward and clockwise with $B$ directed downward. However, orbiting particles have not been observed in the absence of a strong magnetic field in our dc glow discharge setup, indicating that the observations of Ref. 11 may be specific to that experiment.

It is unlikely that ion drag forces drive the motion of the dust grains in our experiment. For ion drag to be responsible for causing the orbiting of the dust particles, the ions would have to $\mathbf{E} \times \mathbf{B}$ drift around a small trapping potential well. However, due to the high ion-neutral collision frequency at neutral pressures of $220 \mathrm{mTorr}\left(\nu_{\text {in }} / \Omega_{i} \approx 12\right)$, the ions cannot complete their gyroorbits and consequently cannot develop an $\mathbf{E} \times \mathbf{B}$ drift. Furthermore, for a radial electric field that can confine charged dust particles, the direction of the ion $\mathbf{E} \times \mathbf{B}$ drift is opposite to that of the cyclotron orbital direction for a negatively charged particle. That is, the ion drag in such a potential well would cause the dust to orbit opposite of the direction expected for negatively charged dust.

Contrary to the experimental observations, the classical Epstein model ${ }^{12}$ of the drag force due to the neutral argon atoms indicates that the orbital motion should be strongly damped for the pressures at which these experiments were conducted. For neutral pressures of the order of 220 mTorr, the neutral drag force is predicted to be $F_{n} \sim 5 \times 10^{-15} \mathrm{~N}$. If the neutral drag force were this large, the particle would not complete an orbit unless there was an azimuthal driving force present. However, consideration of the requirements of such a force makes this possibility unlikely. For example, it is unlikely that there is an azimuthal electric field driving the particle motion since Maxwell's equations would require a monotonic temporal change in the axial magnetic field. Ion drag forces acting in the azimuthal direction are not expected to drive the particles for the reasons discussed above. Radiation pressure on the particle is also ruled out since the orbital motion has been observed with the laser light spread to widths of several centimeters in order to ensure even lighting of the particles over their entire orbit. In addition, to be caused by radiation pressure, the laser light would consistently have to be brighter on the side of the orbit necessary to make the particle rotate in the direction consistent with cyclotron motion (with the field directed upward or downward).

The notion that the neutral drag force may be much smaller than expected is supported by observations. Since the damping rate can be estimated from the resonance width for a damped harmonic oscillator, we can estimate the neutral damping from the width of the spectrum shown in Fig. 3(c). An enlarged view of the normalized spectrum for Particle A, along with spectra calculated for damped harmonic oscillators with different damping rates is shown in Fig. 10. The neutral argon pressure in this case was 220 mTorr. The width of the spectrum indicates that the damping rate is $\sim 0.7 \%$ of the oscillation frequency or $0.0062 \mathrm{~s}^{-1}$. Figure 11 shows a comparison of the resonance widths for two oscillating particles observed at pressures of 220 mTorr and 280 mTorr, respectively. To facilitate comparison of the two spectra, the frequencies have been normalized to the oscillation fre-

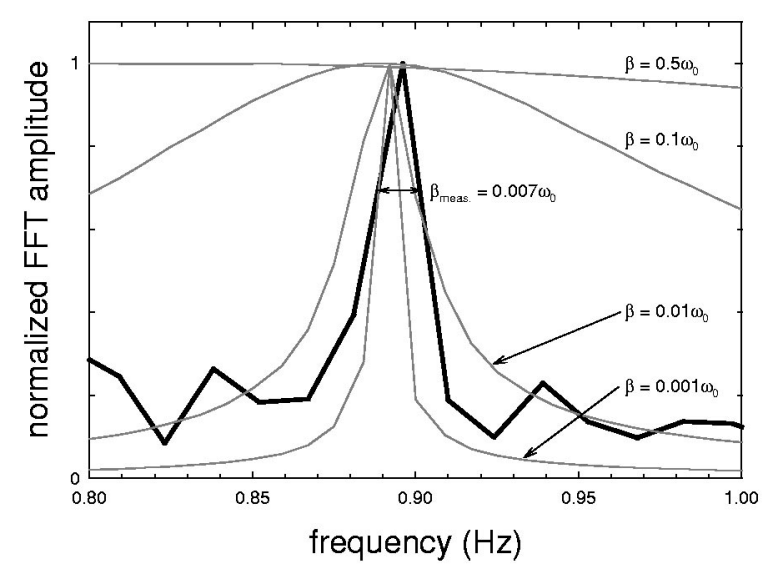

FIG. 10. A comparison of Particle A spectral width to that of damped harmonic oscillators with various damping rates.

quency in each case and the FFT amplitudes have been normalized. At 280 mTorr, the spectral feature is broader than that of the 220 mTorr case. While drawing definitive conclusions regarding neutral drag from these two cases alone is difficult, the data are suggestive that neutral damping increases with pressure, although in each case the neutral drag appears to have much smaller magnitude than predicted by the Epstein model.

Since the observations were made at several different neutral pressures, a comparison of their respective oscillation frequencies can be made. Figure 12 shows a plot of the measured oscillation frequency for the particles included in the analysis for this paper. The frequency is normalized by the cyclotron frequency expected for a $1.2 \mu \mathrm{m}$ particle using the measured values of $\Delta V$ and $B$. The grayed area between the dashed lines indicates the bounds of the expected gyrofrequencies of particles within one standard deviation of the size distribution peak. Although the pressure varies by approximately $30 \%$, the frequency appears to be independent of the neutral gas density. The effects of neutral drag in magnetized dusty plasmas are the focus of a new series of experiments in the DUPLEX device.

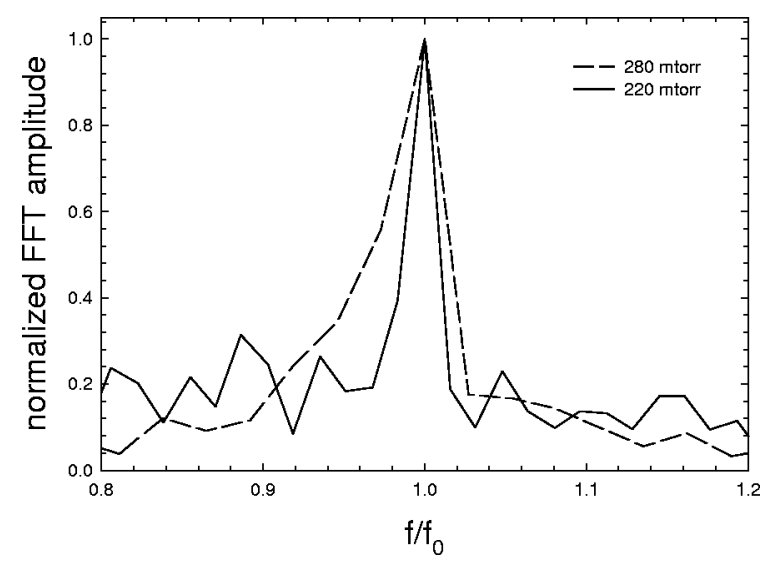

FIG. 11. A comparison of the spectral widths of particle oscillations at 220 mTorr (solid line) and 280 mTorr (dashed line). At 220 mTorr, the $Q$ of the system is approximately 50 , while at 280 mTorr, $Q \sim 25$. 


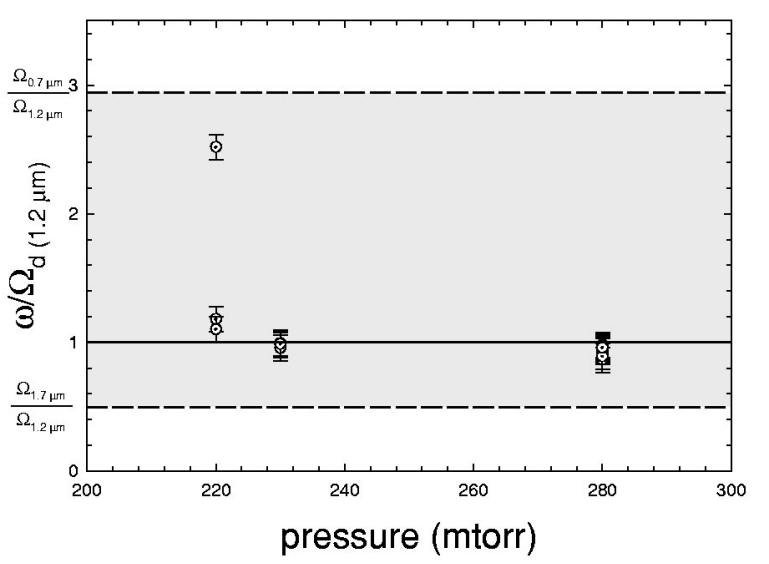

FIG. 12. Normalized oscillation frequency as a function of neutral argon pressure. The data are normalized to the predicted cyclotron frequency for a $1.2 \mu \mathrm{m}$ particle for the ambient plasma conditions and the magnetic field strength. The grayed area indicates the range of values that would be expected for dust particles with diameter within one standard deviation of the peak of the size distribution. The error bars are determined from the spectra of the individual particle position time series.

\section{CONCLUSIONS}

Because of the excellent correspondence between the observed and expected values of frequency and gyroradius, along with rotation in the expected direction about the magnetic field lines, the linear scaling of the rotation frequency and orbital radius, and the close match between the centripetal and magnetic forces, we conclude that the observed particles are magnetized. To our knowledge, these are the first direct observations of such motion. Therefore, these data can be used as a sensitive indicator of the charge on the individual dust grains. The charge can be calculated using the measured particle gyrofrequency, $Q=M \Omega_{d} / B$. For Particle A, this corresponds to a charge $Q=-4.3 \times 10^{-15} \mathrm{C}$, or approximately $2.7 \times 10^{4}$ electrons. Alternately, the charge can be calculated using the expression $Q=4 \pi \varepsilon_{0} r_{d} \Delta V$ using the Langmuir and emissive probe measurements. In this case, corresponds to a charge $Q=-4.7 \times 10^{-15} \mathrm{C}$, or approximately $3.0 \times 10^{4}$ electrons. The charge on Particles B and C are calculated to be $-4.2 \times 10^{-15} \mathrm{C}$ or $\sim 2.64 \times 10^{4}$ electrons and $-3.0 \times 10^{-15} \mathrm{C}$, or approximately $1.9 \times 10^{4}$ electrons, respectively.

There are two distinct advantages of measuring charge by this technique. First, if the particle size is known, then the charge can be found noninvasively, with no knowledge of the plasma parameters required. The charge can simply be calculated using the observed value of gyrofrequency along with the known mass and magnetic field strength and the error in the determined value is limited only by the precision of the mass, magnetic field strength, and gyrofrequency measurements. Once the charge is known, then this technique can also be used to find the local potential relative to the plasma potential by solving the equation $Q=C V$. Using the dust particles themselves as probes is an attractive feature since the technique would be a nonperturbative method for measuring electric field structures with higher spatial resolution than might be acquired with probes.
Second, if the plasma and floating potentials can be measured independently, then for systems with a distribution of particle sizes, measurement of the dust cyclotron frequency offers not only a method for the determination of charge, but can also be useful in determining the size of the observed particle. Equation (1) can be used with the measured oscillation frequency to solve for the dust grain radius $r_{d}$. For example, we find that the diameters of Particles A, B, and C are more likely equal to $1.1 \mu \mathrm{m}, 1.07 \mu \mathrm{m}$, and $0.76 \mu \mathrm{m}$, respectively. Each of these values are well within one standard deviation of the peak of the particle size distribution. This technique may be beneficial in investigations of phenomena relating to space or astrophysical dusty plasmas, which do have distributions of particle sizes and masses and are immersed in magnetic fields. These results also raise the possibility of new experiments to test particle charging theories by varying the magnetic field strength and determining the resulting particle charge.

\section{ACKNOWLEDGMENTS}

We are grateful to Dr. Gurudas Ganguli, Dr. Peter Schuck, Dr. Martin Lampe, Dr. Richard Fernsler, Dr. Leonid Rudakov, Dr. Robert Merlino, Dr. Edward Thomas, Dr. Mihaly Horanyi, Dr. Dan Winske, Dr. Nicola D’Angelo, Dr. Osamu Ishihara, Dr. Norioshi Sato, Dr. Gabriella Paeva, and Dr. Greg Hebner for many useful discussions regarding these observations. The experimental assistance of Mark Cianciosa, Ewen Kelley, and Andrew Straatveit and the assistance of Carl Weber, Doug Mathess, and Tom Milam in the construction of the anode-cathode pair are greatly appreciated.

This work was supported by the Office of Naval Research.

\section{APPENDIX}

In order to independently estimate the charge on a typical dust grain, the floating potential $V_{f}$ and the plasma potential $V_{p}$ were measured using Langmuir and emissive probes at the positions where the particles were found. Figure 13(a) shows a photograph of the discharge plasma with a group of suspended particles visible. (Particle A is also visible as the individual grain to the left of the group of particles.) The image shows that the particles are found near the top of the glow region above the cathode. Near the position of the particles, $V_{f}$ and $V_{p}$ were measured to be $75 \mathrm{~V}$ and $146 \mathrm{~V}$, respectively. The large negative value of $V_{f}-V_{p}$ is indicative of the energetic electrons present in the discharge plasma. In argon, the collision cross section for electrons ${ }^{13}$ with energy on the order of $100 \mathrm{eV}$ is $\sim 3 \times 10^{-16} \mathrm{~cm}^{2}$. At a pressure of 220 mTorr, the mean free path for such energetic electrons is $\sim 0.5 \mathrm{~cm}$. In the presence of energetic electrons, the floating potential of an object immersed in the plasma can attain large negative values with respect to the plasma potential. ${ }^{14}$ In this case, an additional term accounting for the current carried by the primary electrons must be included in the current balance equation when deriving the floating potential: 

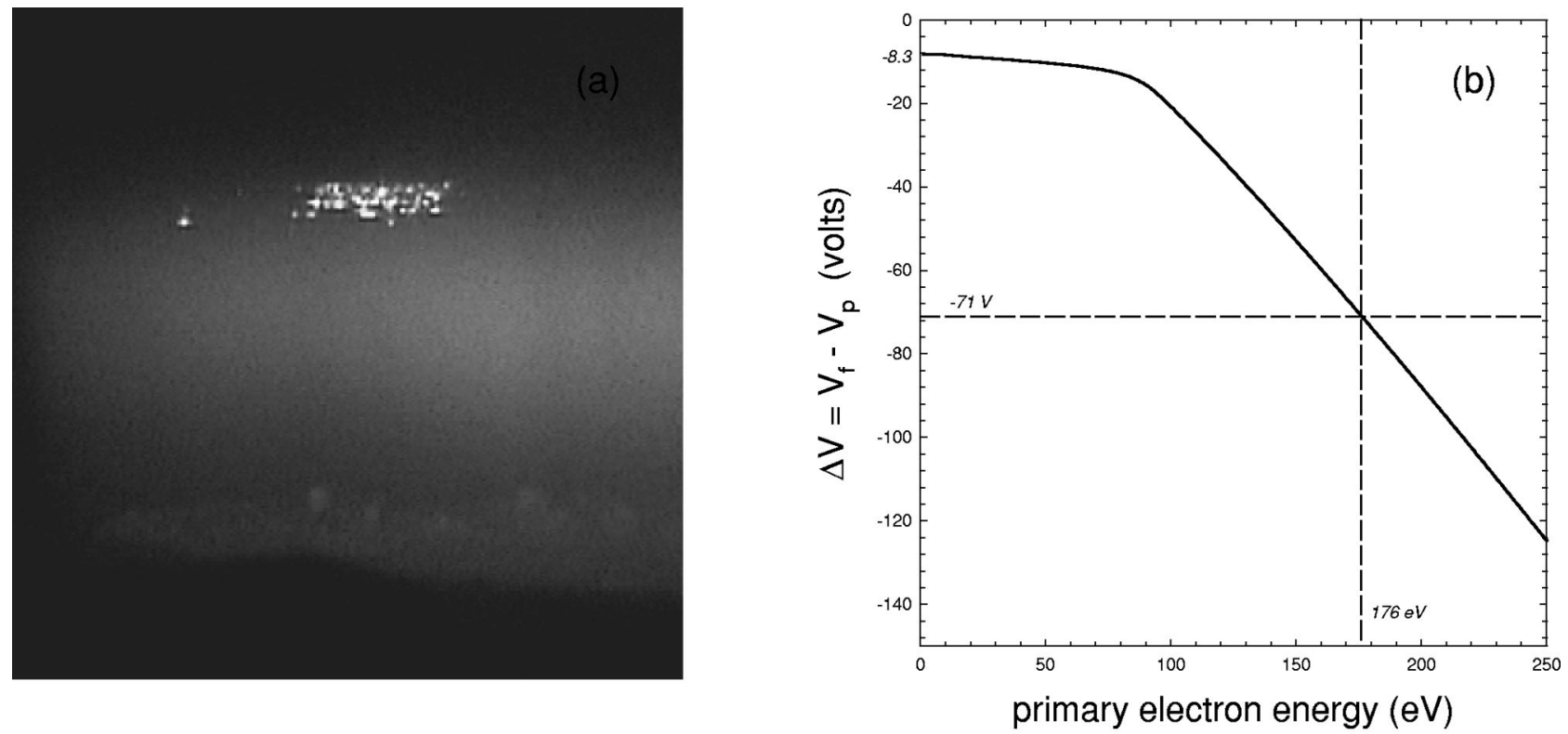

FIG. 13. (a) Photograph of experiment showing levitated particles. Particle A is the individual particle visible to the left of the large group. (b) The predicted value of $\Delta V$ as a function of the primary electron energy $E_{p}$.

$$
\begin{aligned}
I\left(V_{f}\right)= & n e A\left(\frac{k_{\mathrm{B}} T_{e}}{2 \pi m_{e}}\right)^{1 / 2} e^{-\left(e\left(V_{p}-V_{f}\right) / k_{\mathrm{B}} T_{e}\right)} \\
& -0.6 n e A\left(\frac{k_{\mathrm{B}} T_{e}}{m_{i}}\right)^{1 / 2} \\
& +\frac{n_{p} e A}{4}\left(\frac{2 e E_{p}}{m_{e}}\right)^{1 / 2}\left[1-\frac{\left(V_{p}-V_{f}\right)}{E_{p}}\right] \\
= & 0 .
\end{aligned}
$$

Here, the first term represents the plasma electron current, the second term represents the ion current, and the third term represents the current carried by the primary electrons with density $n_{p}$ and energy $E_{p}$. If $n_{p} \rightarrow 0$, Eq. (A1) reduces to the usual form for $V_{f}$. During the experiment, the discharge current was $\sim 5 \mathrm{~mA}$, which is carried by the primary electrons. Assuming that the energy of these electrons is $E_{p} \sim 100 \mathrm{eV}$, this would imply that the primary electron density is $\sim 10^{7} \mathrm{~cm}^{-3}$. Using the measured values of plasma density and the electron temperature, Eq. (A1) can be solved for $\Delta V$. Figure 13(b) shows the calculated value of $\Delta V$ as a function of $E_{p}$. For a beam-to-plasma electron density ratio of $10^{-3}$, a value of $\Delta V=-71 \mathrm{~V}$ implies that the primary electron energy $E_{p}$ is approximately $176 \mathrm{eV}$. Since the discharge voltage was (at least) $220 \mathrm{~V}$ in each of the experiments reported in this paper, and since most of the potential is dropped across the cathode sheath, this estimate is consistent with the value of $\Delta V$ determined from the Langmuir and emissive probe measurements.

${ }^{1}$ B. Walch, M. Horanyi, and S. Robertson, Phys. Rev. Lett. 75, 838 (1995).

${ }^{2}$ A. Homann, A. Melzer, and A. Piel, Phys. Rev. E 59, R3835 (1999).

${ }^{3}$ E. B. Tomme, D. A. Law, B. M. Annaratone, and J. E. Allen, Phys. Rev. Lett. 85, 2518 (2000).

${ }^{4}$ G. A. Hebner, M. E. Riley, D. S. Johnson, P. Ho, and R. J. Buss, Phys. Rev. Lett. 87, 235001 (2001).

${ }^{5}$ N. Sato, T. Mieno, T. Hirata, Y. Yagi, R. Hatakeyama, and S. Iizuka, Phys. Plasmas 1, 3480 (1994).

${ }^{6}$ N. D'Angelo and R. W. Motley, Phys. Fluids 5, 634 (1962).

${ }^{7}$ Q-Z Luo and N. D'Angelo, J. Phys. D 33, 2754 (2000).

${ }^{8}$ If the data point $(0,0)$ is added to the measurements, indicating that the rotation frequency is 0 with no applied magnetic field, the slope of the fitted line is found to yield $Q / M=12.1$. In all of our previous experiments, performed under similar setups and conditions but without a magnetic field, orbiting particles were not observed.

${ }^{9}$ N. Sato, G. Uchida, R. Ozaki, S. Iizuka, and T. Kamimura, Frontiers in Dusty Plasmas, edited by Y. Nakamura, T. Yokota, and P. K. Shukla (Elsevier Science, New York, 2000), p. 329.

${ }^{10}$ O. Ishihara and N. Sato, IEEE Trans. Plasma Sci. 29, 179 (2001).

${ }^{11}$ G. Paeva, R. P. Dahiya, G. M. W. Kroesen, and W. W. Stoffels, "Rotation of particles trapped in the sheath of a radio-frequency capacitively coupled plasma," IEEE Trans. Plasma Sci. (to be published).

${ }^{12}$ P. S. Epstein, Phys. Rev. 23, 710 (1924).

${ }^{13}$ R. D. DuBois and M. J. Rudd, J. Phys. B 8, 1474 (1975).

${ }^{14}$ N. Hershkowitz, "How Langmuir probes work," Plasma Diagnostics, edited by O. Auciello and D. Flamm (Academic, New York, 1989), p. 113. 